\title{
Preferential Oxidative Addition in Suzuki Cross-Coupling Reactions across one Fluorene Unit
}

\author{
Sven K. Weber, Frank Galbrecht and Ullrich Scherf
}

Makromolekulare Chemie and Institut für Polymertechnologie, Bergische Universität Wuppertal, Gauss-Str. 20, D-42097 Wuppertal, Germany

scherf@uni-wuppertal.de

\section{Supporting Information}

\section{Table of contents}

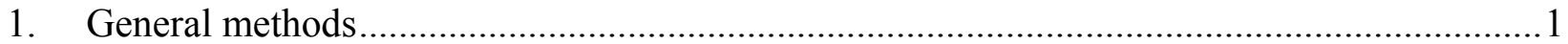

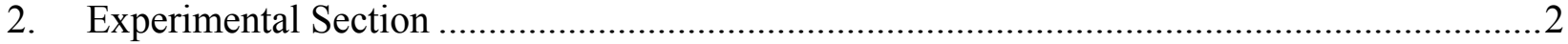

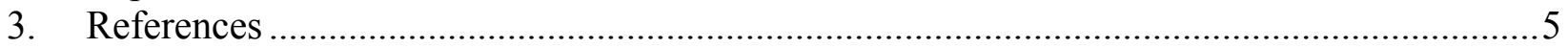

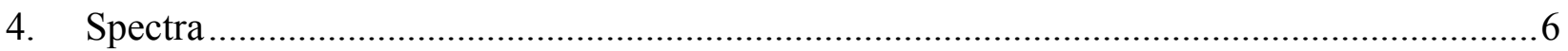

\section{General methods}

Unless otherwise indicated, all starting materials were purchased from commercial sources (Aldrich, Fischer, EM Science, Lancaster, ABCR, Strem) and used without further purification. All reactions were assembled under an inert atmosphere either in a screw capped vial or in a resealable Schlenk tube. ${ }^{1} \mathbf{H}$ - and ${ }^{13} \mathbf{C}$-NMR spectra were recorded on a Bruker ARX 400spectrometer. Chemical shifts $(\delta)$ are reported in parts per million (ppm) using residual solvents protons as internal standards. The coupling constants are reported in Hertz (Hz). Splitting patterns are designated as s (singlet), d (doublet), $\mathrm{t}$ (triplet), q (quartet), bs (broad singlet), $\mathrm{m}$ (multiplet) and bm (broad multiplet). Low resolution mass spectrometry was obtained on a Varian MAT 311A operating at $70 \mathrm{eV}$ (Electron Impact, EI) and reported as $\mathrm{m} / \mathrm{z}$ and percent 
relative intensity. GC/MS data were recorded on a Shimadzu GC-17a and Shimadzu GCMSQP5050 (Column: FS-OV1-CB-0,25) and reported listing the retention time in min., the observed molecular weight and total percent. Helium was the carrier gas. The following conditions were used for all GC-MS analyses: Injector temperature: $280{ }^{\circ} \mathrm{C}$, initial temperature: $250{ }^{\circ} \mathrm{C}$, temperature rate: $6{ }^{\circ} \mathrm{C} \mathrm{min}^{-1}$, final temperature: $280{ }^{\circ} \mathrm{C}$, final time: $30 \mathrm{~min}$.

\section{Experimental Section}

\subsection{2,7-Dibromo-9,9'-bis(2-ethylhexyl)fluorene (4a) ${ }^{1}$}

To a solution of 2,7-dibromofluorene $(33.0 \mathrm{~g}, 102 \mathrm{mmol})$ and tetrabutylammonium bromide (9.9 g, $31 \mathrm{mmol})$ in DMSO (75 mL) aqueous sodium hydroxide solution (40 mL, 50\%) and (2ethylhexyl)bromide (43.3 g, $225 \mathrm{mmol})$ were added. The mixture was stirred at $80{ }^{\circ} \mathrm{C}$ for $2 \mathrm{~h}$ and then poured into water $(100 \mathrm{~mL})$. The mixture was extracted two times with diethyl ether and the combined organic phases were washed with brine, water and dried over $\mathrm{Na}_{2} \mathrm{SO}_{4}$. Upon evaporating off the solvent the residue was purified via column chromatography with $n$-hexane as eluent to afford a colourless oil, which was recrystallized from ethanol at $-20{ }^{\circ} \mathrm{C}$ to afford colourless crystals $(76 \%)$.

${ }^{1} \mathrm{H}$ NMR $\left(400 \mathrm{MHz}, \mathrm{C}_{2} \mathrm{D}_{2} \mathrm{Cl}_{4}, 25{ }^{\circ} \mathrm{C}\right): \delta=7.53-7.39(\mathrm{~m}, 6 \mathrm{H}, \mathrm{Ar}-\mathrm{H}), 1.91(\mathrm{~d}, 4 \mathrm{H}, J=5.7 \mathrm{~Hz}, \mathrm{Ar}-$ $\left.\mathrm{CH}_{2}\right), 1.00-0.35\left(\mathrm{~m}, 30 \mathrm{H}\right.$, Alkyl-H) ppm. ${ }^{13} \mathrm{C} \mathrm{NMR}\left(100 \mathrm{MHz}, \mathrm{C}_{2} \mathrm{D}_{2} \mathrm{Cl}_{4}, 25{ }^{\circ} \mathrm{C}\right): \delta=152.4$, 139.1, 130.0, 127.4, 121.0, 120.9, 55.3, 44.3, 34.7, 33.5, 28.0, 27.0, 22.7, 13.9, 10.3 ppm. LR-MS (EI, m/z): 41 (23.7), 43 (40.6), 57 (100.0), 71 (29.5), 141 (17.0), 176 (11.6), 546 (23.8), 548 [M ] (45.7), 549 (14.2), 550 (23.8).

\subsection{2,7-Diiodo-9,9'-bis(2-ethylhexyl)fluorene (4b) ${ }^{2}$}

2,7-Diiodofluorene $(10.0 \mathrm{~g}, 24 \mathrm{mmol})$ was dissolved in THF and treated with potassium tertbutoxide $(3.0 \mathrm{~g}, 27 \mathrm{mmol})$ to give a red solution, followed by alkylation with (2-ethylhexyl)bromide $(3.8 \mathrm{~g}, 27 \mathrm{mmol})$. This procedure was repeated once more. The product was purified by column chromatography using $n$-hexane as an eluent to yield $8.0 \mathrm{~g}(75 \%)$ of a yellow solid with purity greater than $99 \%$ detected by GC-MS. 
${ }^{1} \mathrm{H}$ NMR (400 MHz, $\left.\mathrm{C}_{2} \mathrm{D}_{2} \mathrm{Cl}_{4}, 25{ }^{\circ} \mathrm{C}\right): \delta=7.33-7.63$ (m, $\left.6 \mathrm{H}, \mathrm{Ar}-\mathrm{H}\right), 1.78-1.87\left(\mathrm{~m}, 4 \mathrm{H}, \mathrm{Ar}-\mathrm{CH}_{2}\right)$, 0.60-0.84 (m, 18H, Alkyl-H), 0.66 (t, 6H, $\left.-\mathrm{CH}_{3}, J=6.8 \mathrm{~Hz}\right), 0.47$ (dt, 6H, $-\mathrm{CH}_{3}, J=1.9 \mathrm{~Hz}$, $J=7.2 \mathrm{~Hz}) \mathrm{ppm} .{ }^{13} \mathrm{C} \mathrm{NMR}\left(100 \mathrm{MHz}, \mathrm{C}_{2} \mathrm{D}_{2} \mathrm{Cl}_{4}, 25^{\circ} \mathrm{C}\right): \delta=152.6,140.1,136.1,133.6,127.5$, 121.7, 92.7, 55.4, 44.3, 34.9, 28.3, 27.4, 23.0, 14.5, 10.7 ppm. LR-MS (EI, m/z): 41 (28.4), 43 (46.3), 57 (100.0), 71 (18.6), 176 (13.9), 304 (12.4), 402 (21.8), 416 (10.0), 430 (11.6), 642 [M ] (96.1). GC-MS (retention time, $\mathrm{M}_{\mathrm{w}}$ obsd, \% Total): $7.7 \mathrm{~min}, 642\left(\mathrm{C}_{29} \mathrm{H}_{40} \mathrm{I}_{2}\right), 99.3 \%$.

\section{General Procedure for Suzuki Cross-Coupling Reactions}

The boronic acid (1 eq.), $\mathrm{Pd}_{2}(\mathrm{dba})_{3}, \mathrm{P}(t-\mathrm{Bu})_{3}$ and $\mathrm{K}_{3} \mathrm{PO}_{4}$ (3 eq.) were added to a screw capped vial or a Schlenk tube equipped with a stir bar. The tube was fitted with a rubber septum, evacuated, and then refilled with argon. Dry THF was added via syringe. Three freeze-pumpthaw-cycles were performed. Then the solution was allowed to stir for 5-10 min. The dihalide (1 eq.) was added in a second Schlenk tube or a screw capped vial equipped with a stir bar. This was fitted with a rubber septum, evacuated, and then refilled with argon. Dry THF was added via syringe. Three freeze-pump-thaw-cycles were performed with the dihalide solution. Then the dihalide solution was added to the solution of the first tube via a double-ended needle. Three freeze-pump-thaw-cycles were performed again. The mixture was stirred at the indicated temperature for the indicated amount of time. At the end of the reaction, the mixture was quenched with water, diluted with ethyl acetate and filtered. The organic layer was washed with $2 \mathrm{M} \mathrm{HCl}$, saturated aqueous $\mathrm{NaHCO}_{3}$ and EDTA solutions and dried over $\mathrm{Na}_{2} \mathrm{SO}_{4}$. The solvent was removed via rotary evaporation.

\subsection{2,7-Bisphenyl-9,9'-bis(2-ethylhexyl)fluorene (12a) ${ }^{3}$}

The general procedure was followed, using 2,7-dibromo-9,9'-bis(2-ethylhexyl)fluorene (4a) (548.4 mg, $1 \mathrm{mmol})$, benzeneboronic acid (121.9 mg, $1 \mathrm{mmol}) \mathrm{Pd}_{2}(\mathrm{dba})_{3}(13.8 \mathrm{mg}, 0.015 \mathrm{mmol})$, $\mathrm{P}(t-\mathrm{Bu})_{3}(12.1 \mathrm{mg}, 0.06 \mathrm{mmol})$ and $\mathrm{K}_{3} \mathrm{PO}_{4}(690.8 \mathrm{mg}, 3 \mathrm{mmol})$ allowed to react for 5 days at $80{ }^{\circ} \mathrm{C}$. Column chromatography ( silica gel, hexane, Rf: 0.29) enabled isolation of the pure disubstituted product as a yellow oil (89\%). 
GC-MS (retention time, $\mathrm{M}_{\mathrm{w}}$ obsd, \% Total): $5.4 \mathrm{~min}, 548\left(\mathrm{C}_{29} \mathrm{H}_{40} \mathrm{Br}_{2}\right.$, starting material), 52.0\%; $19.0 \mathrm{~min}, 542\left(\mathrm{C}_{41} \mathrm{H}_{50}, \mathrm{di}\right), 48.0 \%$. ${ }^{1} \mathrm{H}$ NMR (400 MHz, $\left.\mathrm{C}_{2} \mathrm{D}_{2} \mathrm{Cl}_{4}, 25{ }^{\circ} \mathrm{C}\right): \delta=7.77(\mathrm{~d}, 2 \mathrm{H}$, $J=7.8 \mathrm{~Hz}, \mathrm{Ar}-\mathrm{H}), 7.56-7.66(\mathrm{~m}, 8 \mathrm{H}, \mathrm{Ar}-\mathrm{H}), 7.47$ (t, 4H, J=7.6 Hz, Ar-H), 7.36 (t, 2H, $J=7.4 \mathrm{~Hz}, \mathrm{Ar}-\mathrm{H}), 2.03-2.13\left(\mathrm{~m}, 4 \mathrm{H}, \mathrm{Ar}-\mathrm{CH}_{2}\right), 0.77-1.01\left(\mathrm{~m}, 18 \mathrm{H},-\mathrm{CH}_{2}\right), 0.61-0.64(\mathrm{~m}, 6 \mathrm{H}$, $\left.\mathrm{CH}_{3}\right), 0.52-0.57\left(\mathrm{~m}, 6 \mathrm{H},-\mathrm{CH}_{3}\right)$ ppm. ${ }^{13} \mathrm{C} \mathrm{NMR}\left(100 \mathrm{MHz}, \mathrm{C}_{2} \mathrm{D}_{2} \mathrm{Cl}_{4}, 25{ }^{\circ} \mathrm{C}\right): \delta=151.2,141.9$, $140.3,139.5,128.7,127.2,126.9,126.1,122.8,119.8,55.1,44.6,34.7,33.9$, 28.2, 27.1, 22.7, 13.9, 10.3 ppm. LR-MS (EI, m/z): 41 (29.7), 43 (45.2), 55 (17.7), 57 (66.6), 71 (17.5), 149 (15.6), 169 (11.0), 317 (22.9), 330 (34.8), 332 (23.4), $542\left[\mathrm{M}^{+}\right](100.0)$.

\subsection{2,7-Bis(4-tert-butylphenyl)-9,9'-bis(2-ethylhexyl)fluorene (12b)}

The general procedure was followed, using 2,7-dibromo-9,9'-bis(2-ethylhexyl)fluorene (4a) (274.2 mg, $0.5 \mathrm{mmol})$, 4-tert-butylbenzeneboronic acid (89.0 mg, $0.5 \mathrm{mmol}) \mathrm{Pd}_{2}(\mathrm{dba})_{3}(6.9 \mathrm{mg}$, $0.008 \mathrm{mmol}), \mathrm{P}(t-\mathrm{Bu})_{3}(6.1 \mathrm{mg}, 0.03 \mathrm{mmol})$ and $\mathrm{K}_{3} \mathrm{PO}_{4}(345.4 \mathrm{mg}, 1.5 \mathrm{mmol})$ allowed to react for 5 days at $80^{\circ} \mathrm{C}$.

GC-MS (retention time, $\mathrm{M}_{\mathrm{w}}$ obsd, \% Total): $2.8 \mathrm{~min}, 390\left(\mathrm{C}_{29} \mathrm{H}_{40}\right.$, dehalogenated starting material), 9.0\%; 3.9 min, $468\left(\mathrm{C}_{29} \mathrm{H}_{40} \mathrm{Br}\right.$, dehalogenated starting material), $11.3 \%$; $5.4 \mathrm{~min}$, $548\left(\mathrm{C}_{29} \mathrm{H}_{40} \mathrm{Br}_{2}\right.$, starting material), $79.0 \% ; 25.7 \mathrm{~min}, 392\left(\mathrm{C}_{41} \mathrm{H}_{50}, \mathrm{di}\right), 0.7 \%$.

\subsection{2,7-Diphenyl-9,9'-bis(2-ethylhexyl)fluorene (12a) ${ }^{3}$}

The general procedure was followed, using 2,7-diiodo-9,9'-bis(2-ethylhexyl)fluorene $(4, \mathrm{X}=\mathrm{I})$ (321.2 $\mathrm{mg}, \quad 0.5 \mathrm{mmol})$, benzeneboronic acid (61.0 mg, $0.5 \mathrm{mmol}) \quad \mathrm{Pd}_{2}(\mathrm{dba})_{3} \quad(6.9 \mathrm{mg}$, $0.008 \mathrm{mmol}), \mathrm{P}(t-\mathrm{Bu})_{3}(6.1 \mathrm{mg}, 0.03 \mathrm{mmol})$ and $\mathrm{K}_{3} \mathrm{PO}_{4}(345.4 \mathrm{mg}, 1.5 \mathrm{mmol})$ allowed to react for 5 days at $80^{\circ} \mathrm{C}$. Column chromatography (silica gel, hexane, Rf: 0.29) enabled isolation of the pure disubstituted product as a yellow oil (40\%).

GC-MS (retention time, $\mathrm{M}_{\mathrm{w}}$ obsd, \% Total): $5.5 \mathrm{~min}, 390\left(\mathrm{C}_{29} \mathrm{H}_{40}\right.$, dehalogenated starting material), 5.5\%; $4.5 \mathrm{~min}, 516\left(\mathrm{C}_{29} \mathrm{H}_{40} \mathrm{I}\right.$, dehalogenated starting material), $7.7 \%$; $5.6 \mathrm{~min}$, $642\left(\mathrm{C}_{29} \mathrm{H}_{40} \mathrm{I}_{2}\right.$, starting material), 33.3\%; $19.2 \mathrm{~min}, 542\left(\mathrm{C}_{41} \mathrm{H}_{50}, \mathrm{di}\right), 53.5 \% .{ }^{1} \mathrm{H}$ NMR (400 MHz, $\left.\mathrm{C}_{2} \mathrm{D}_{2} \mathrm{Cl}_{4}, 25^{\circ} \mathrm{C}\right): \delta=7.77-7.79(\mathrm{~m}, 2 \mathrm{H}, \mathrm{Ar}-\mathrm{H}), 7.58-7.67(\mathrm{~m}, 8 \mathrm{H}, \mathrm{Ar}-\mathrm{H}), 7.48(\mathrm{t}, 4 \mathrm{H}, J=7.6 \mathrm{~Hz}$, 
$\operatorname{Ar}-\mathrm{H}), 7.37$ (t, $2 \mathrm{H}, J=7.3 \mathrm{~Hz}, \mathrm{Ar}-\mathrm{H}), 2.05-2.15\left(\mathrm{~m}, 4 \mathrm{H}, \mathrm{Ar}-\mathrm{CH}_{2}\right), 0.76-1.02\left(\mathrm{~m}, 18 \mathrm{H},-\mathrm{CH}_{2}\right)$, 0.64-0.66 (m, 6H, - $\left.\mathrm{CH}_{3}\right), 0.54-0.59\left(\mathrm{~m}, 6 \mathrm{H},-\mathrm{CH}_{3}\right) \mathrm{ppm} .{ }^{13} \mathrm{C} \mathrm{NMR}\left(100 \mathrm{MHz}, \mathrm{C}_{2} \mathrm{D}_{2} \mathrm{Cl}_{4}, 80{ }^{\circ} \mathrm{C}\right): \delta$ $=151.2,141.9,140.3,139.5,128.7,127.2,126.9,126.1,122.8,119.8,55.1,44.6,34.7,33.9$, 28.2, 27.1, 22.7, 13.9, 10.3 ppm. LR-MS (EI, m/z): 41 (30.6), 43 (54.5), 55 (14.4), 57 (85.3), 71 (20.1), 149 (13.9), 317 (35.5), 318 (10.0), 330 (32.9), 331 (26.6), $542\left[\mathrm{M}^{+}\right](100.0), 543$ (45.0).

\subsection{2,7-Bis(4-tert-butylphenyl)-9,9'-bis(2-ethylhexyl)fluorene (12b)}

The general procedure was followed, using 2,7-diiodo-9,9'-bis(2-ethylhexyl)fluorene (4, X = I) (321.2 mg, $0.5 \mathrm{mmol})$, 4-tert-butylboronic acid $(89.0 \mathrm{mg}, 0.5 \mathrm{mmol}) \quad \mathrm{Pd}_{2}(\mathrm{dba})_{3} \quad(6.9 \mathrm{mg}$, $0.008 \mathrm{mmol}), \mathrm{P}(t-\mathrm{Bu})_{3}(6.1 \mathrm{mg}, 0.03 \mathrm{mmol})$ and $\mathrm{K}_{3} \mathrm{PO}_{4}(345.4 \mathrm{mg}, 1.5 \mathrm{mmol})$ allowed to react for 5 days at $80^{\circ} \mathrm{C}$.

GC-MS (retention time, $\mathrm{M}_{\mathrm{w}}$ obsd, \% Total): $2.9 \mathrm{~min}, 390\left(\mathrm{C}_{29} \mathrm{H}_{40}\right.$, dehalogenated starting material), 51.4\%; $4.7 \mathrm{~min}, 516\left(\mathrm{C}_{29} \mathrm{H}_{40} \mathrm{I}\right.$, dehalogenated starting material), $48.6 \%$.

\section{References}

(1) Woo, E. P.; Inbasekaran, M.; Shiang, W. R.; Roof, G. R.; WO97/05184, 1997.

(2) Okumoto, K.; Shirota, Y. Chem. Mater. 2003, 15, 699.

(3) Ma, W.; Wu, Y.; Gu, D.; Gan, F.; CN 1587252, 2005. 
4. Spectra

\subsection{2,7-Dibromo-9,9'-bis(2-ethylhexyl)fluorene (4a)}

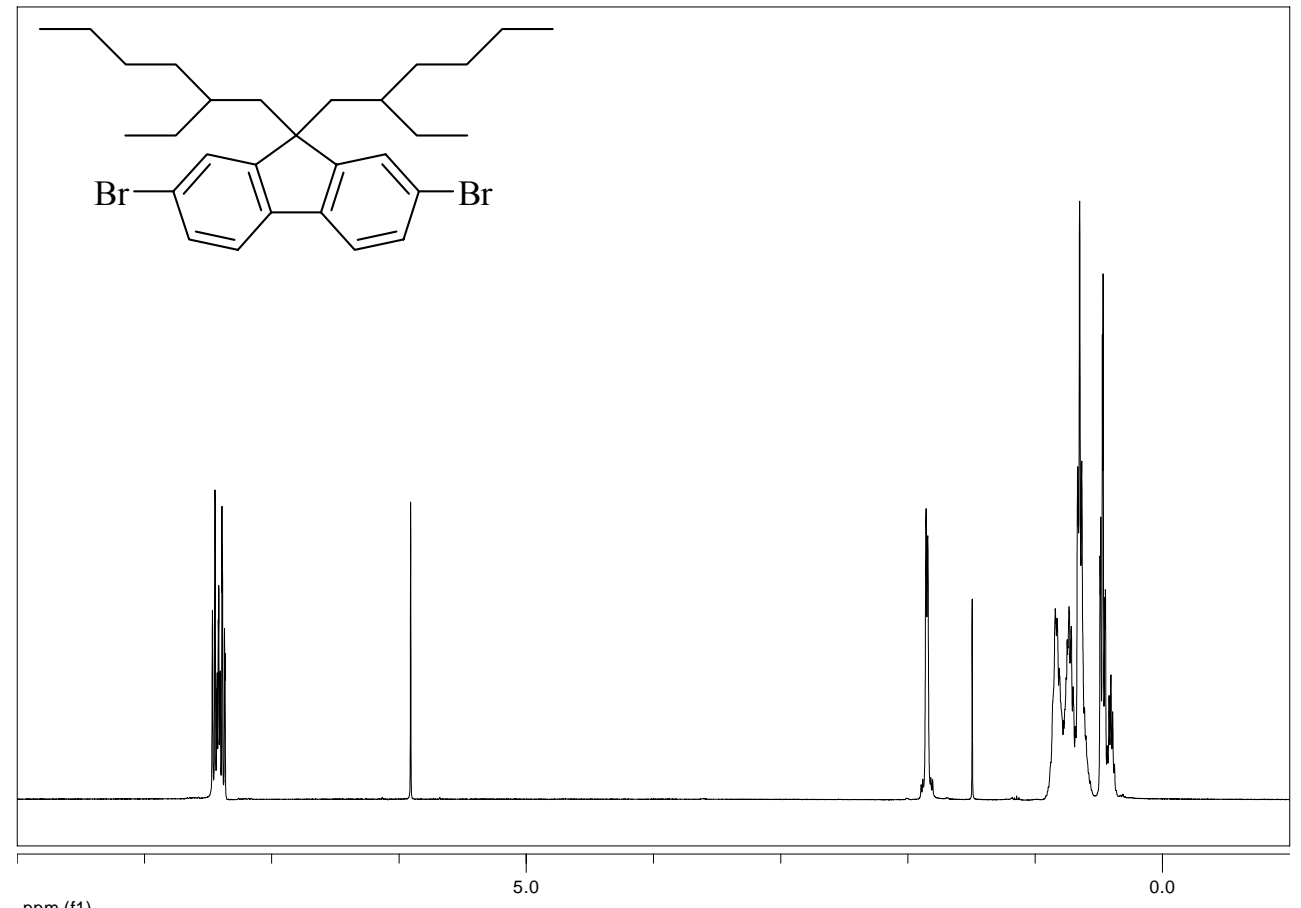

Figure 1 - ${ }^{1} \mathrm{H}$-NMR of 2,7-Dibromo-9,9'-bis(2-ethylhexyl)fluorene (4a).

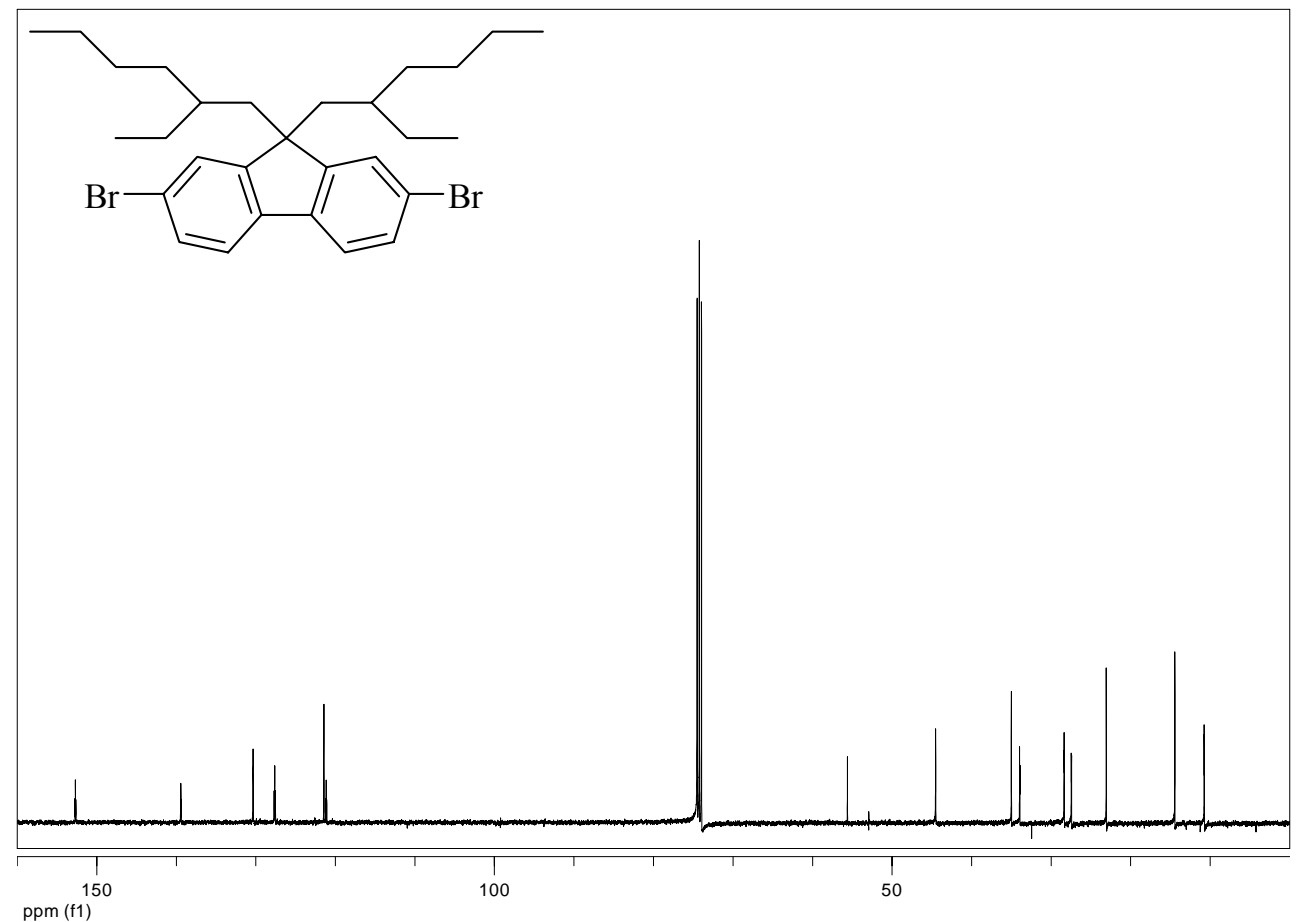

Figure 2 - ${ }^{13}$ C-NMR of 2,7-Dibromo-9,9'-bis(2-ethylhexyl)fluorene (4a). 


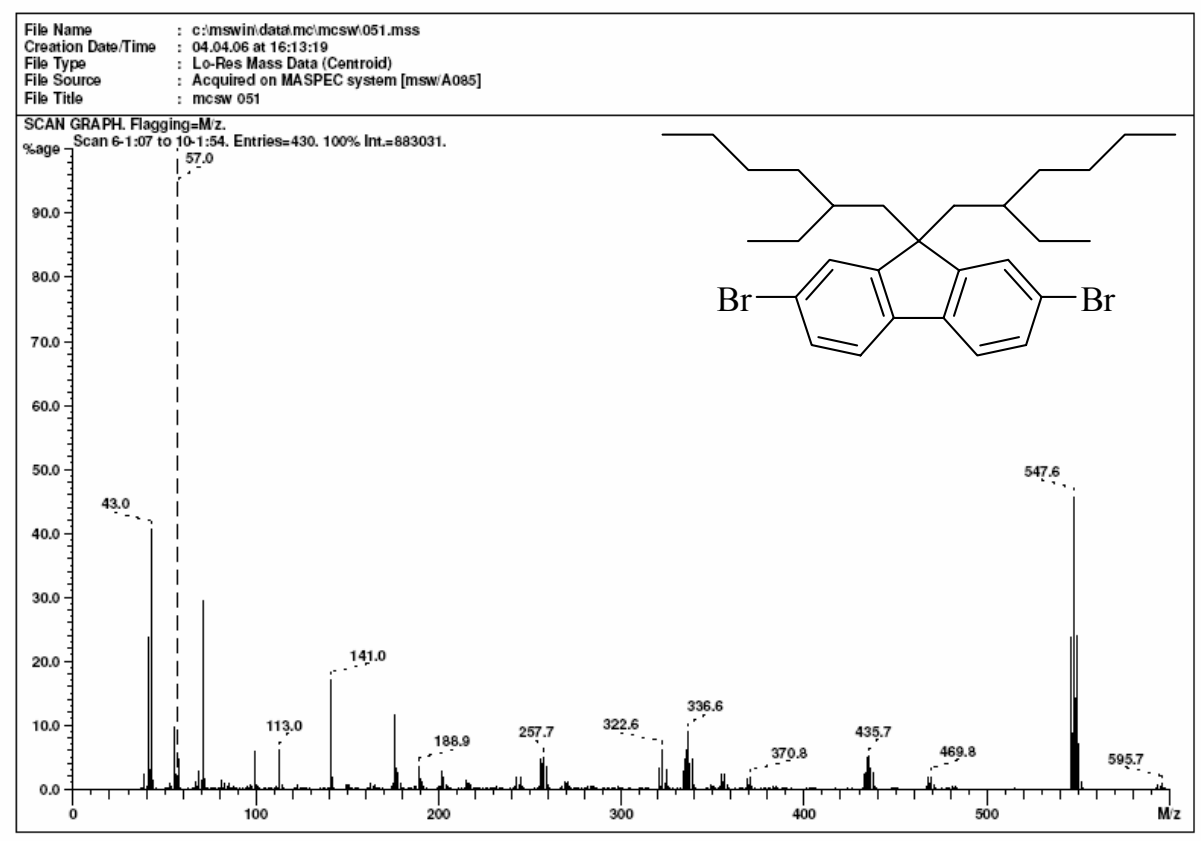

Figure 3 -Mass spectra of 2,7-Dibromo-9,9'-bis(2-ethylhexyl)fluorene (4a).

\subsection{2,7-Diiodo-9,9'-bis(2-ethylhexyl)fluorene (4b)}

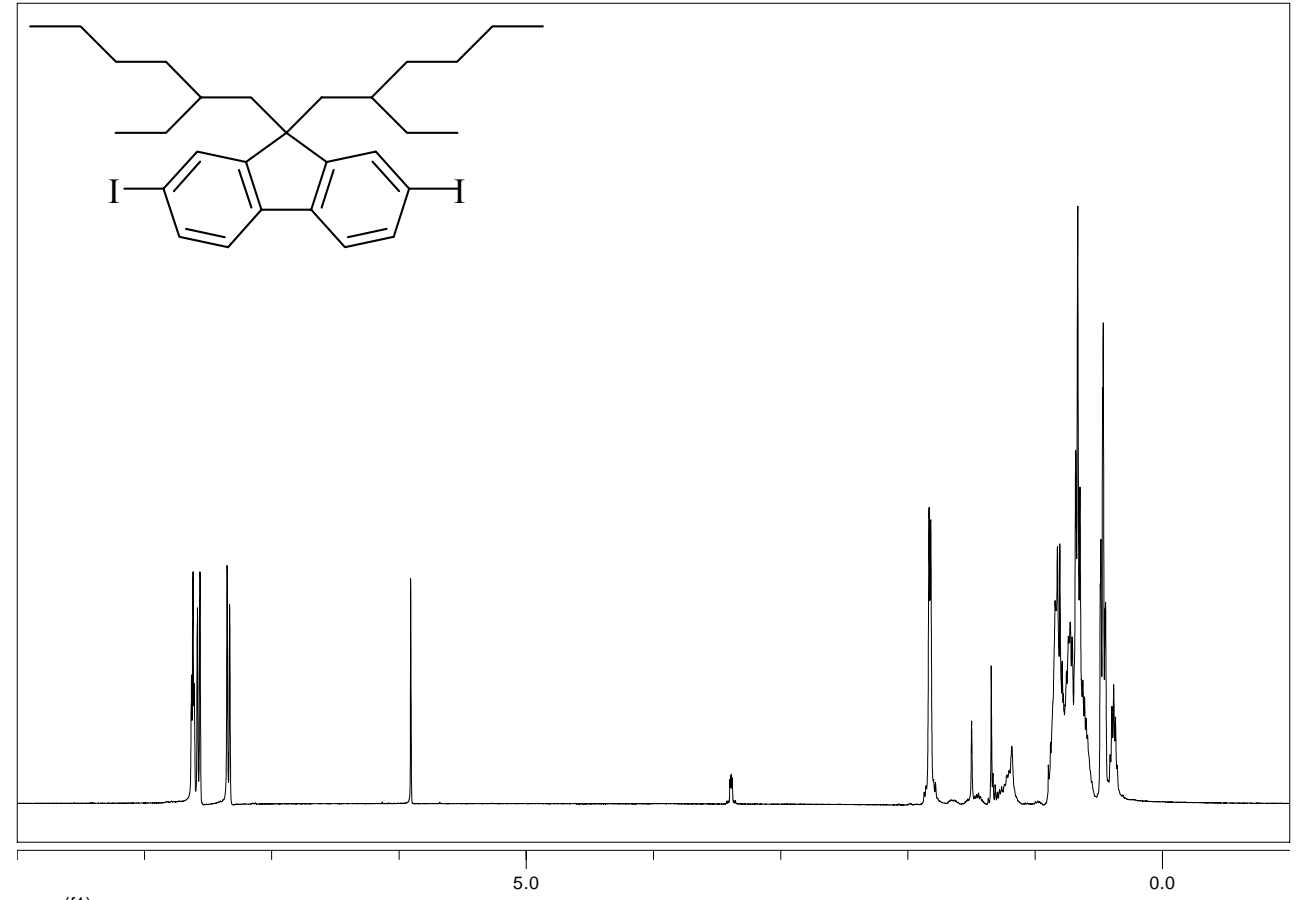

ppm (f1)

Figure 4 - ${ }^{1} \mathrm{H}$-NMR of 2,7-Diiodo-9,9'-bis(2-ethylhexyl)fluorene (4b). 


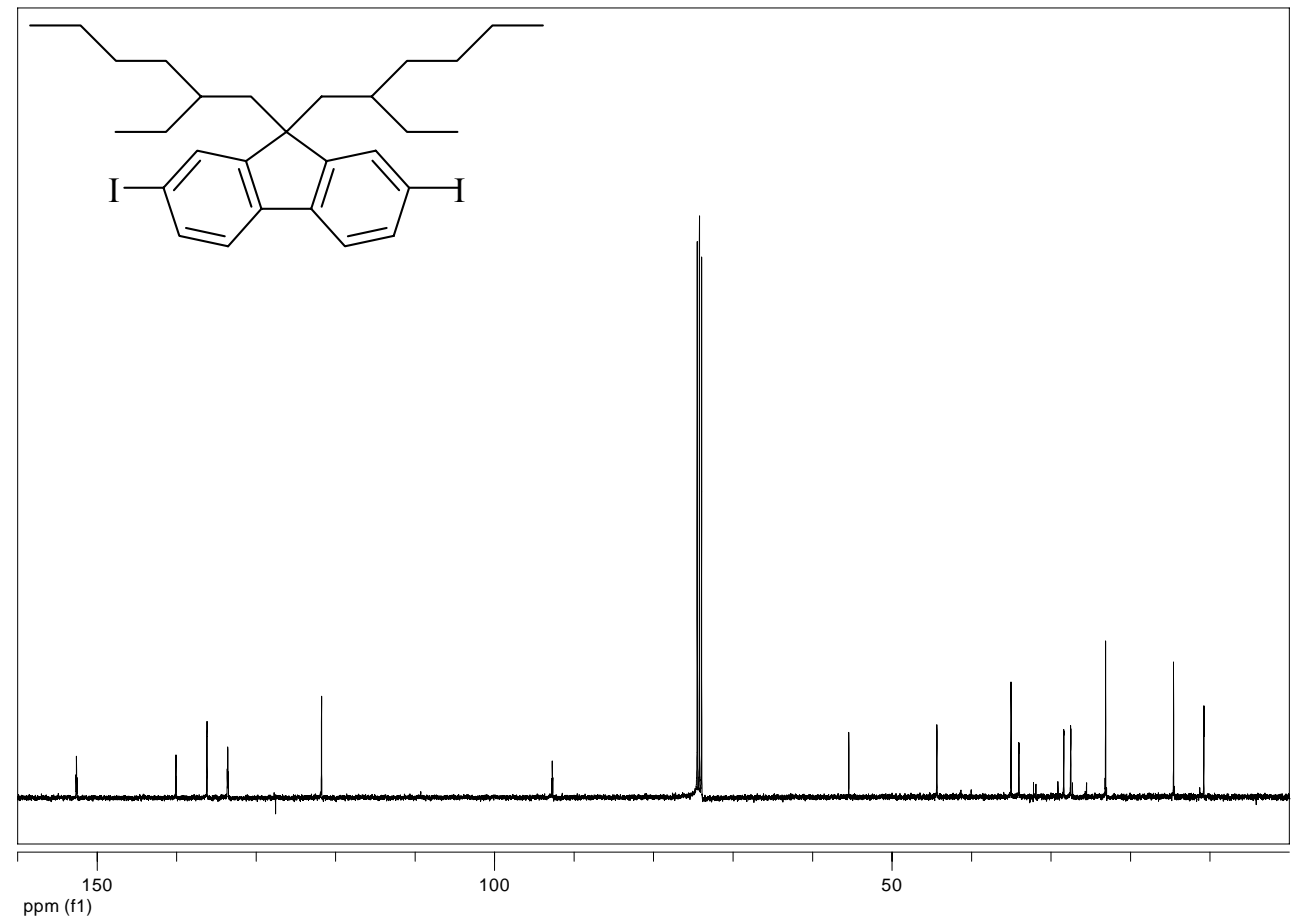

Figure 5 - ${ }^{13}$ C-NMR of 2,7-Diiodo-9,9'-bis(2-ethylhexyl)fluorene (4b).

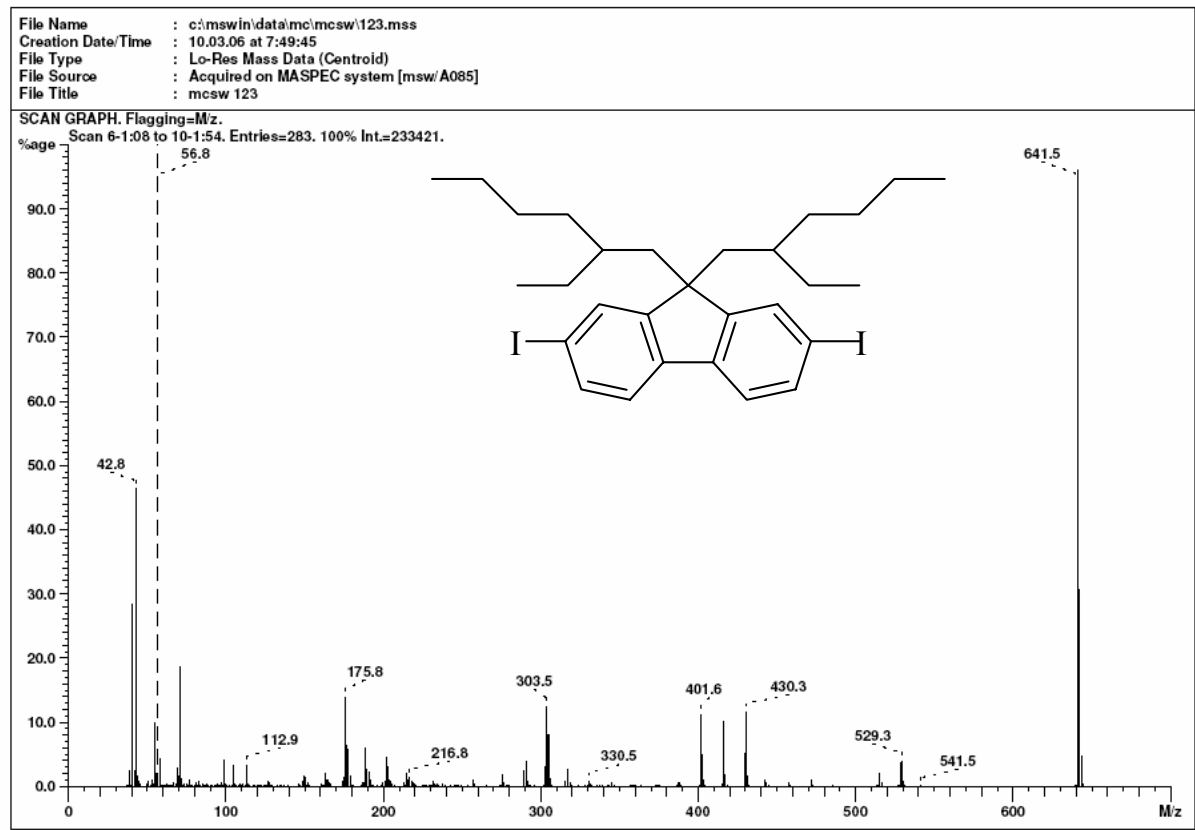

Figure 6 - Mass spectra of 2,7-Diiodo-9,9'-bis(2-ethylhexyl)fluorene (4b). 


\subsection{2,7-Bisphenyl-9,9'-bis(2-ethylhexyl)fluorene (12a, Table 1, Entry 1)}
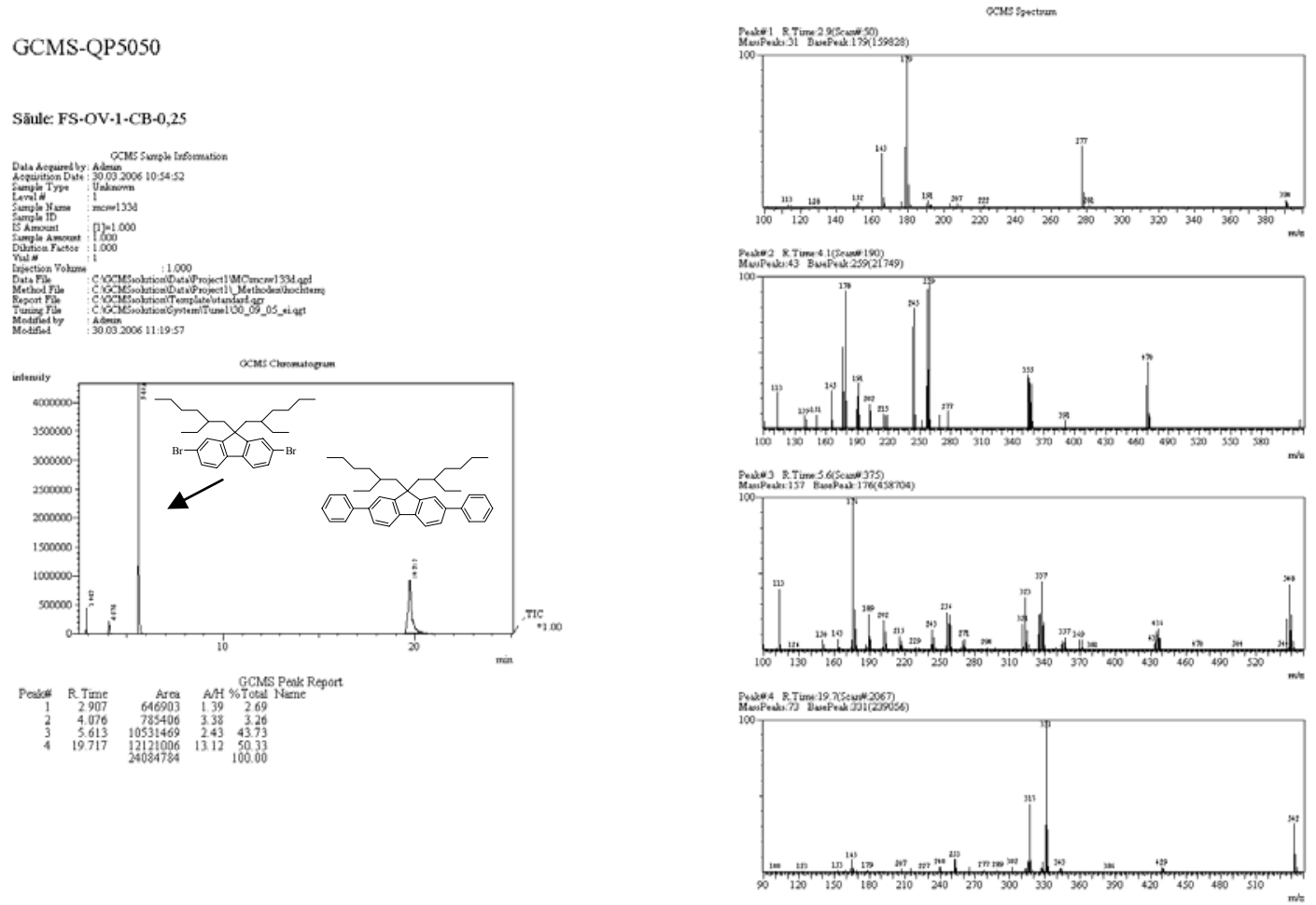

Figure 7 - GC-MS spectra of entry 1 (Mixture of 4a and 12a).

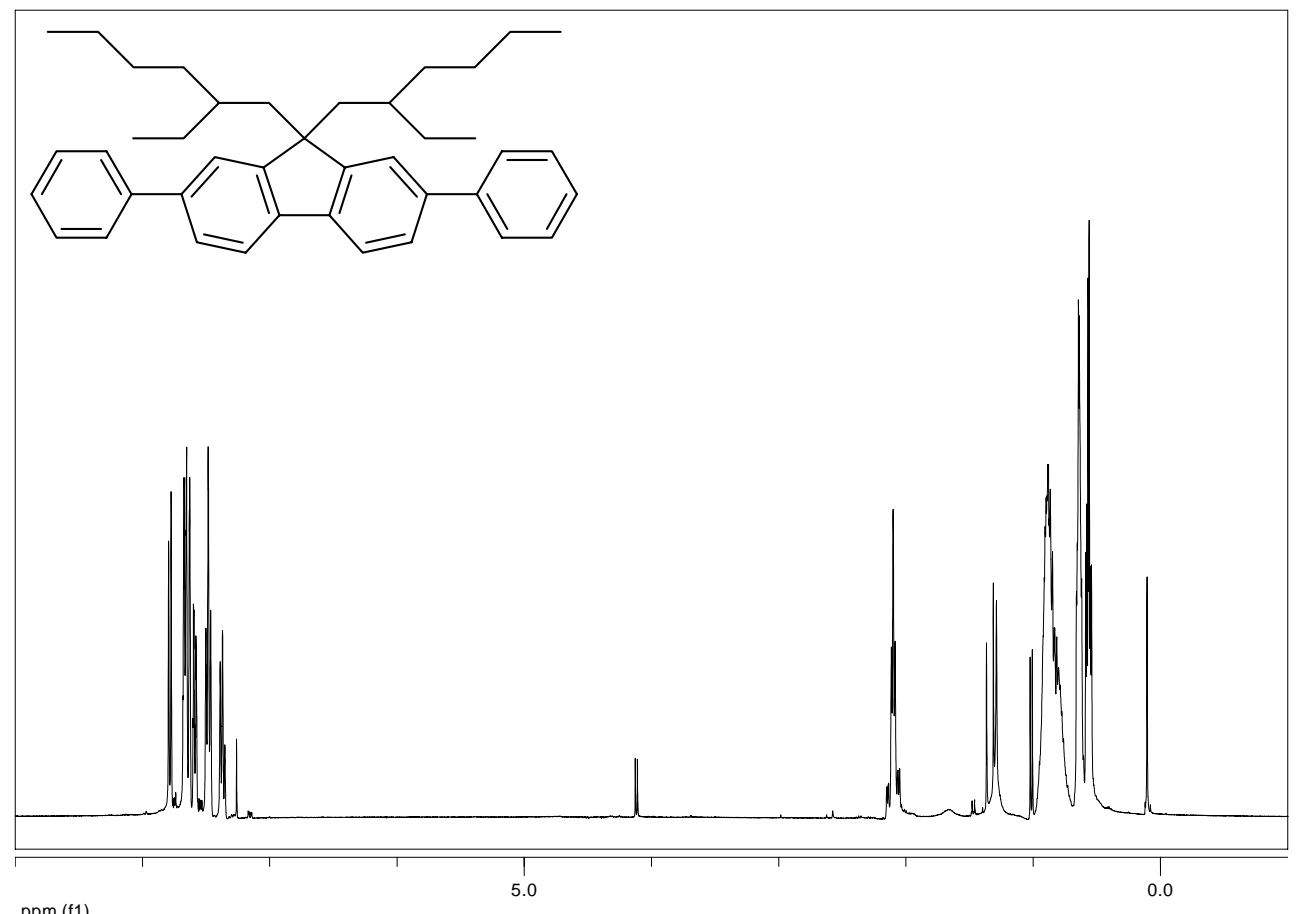

Figure 8 - ${ }^{1}$ H-NMR of 2,7-Bisphenyl-9,9'-bis(2-ethylhexyl)fluorene (12a). 


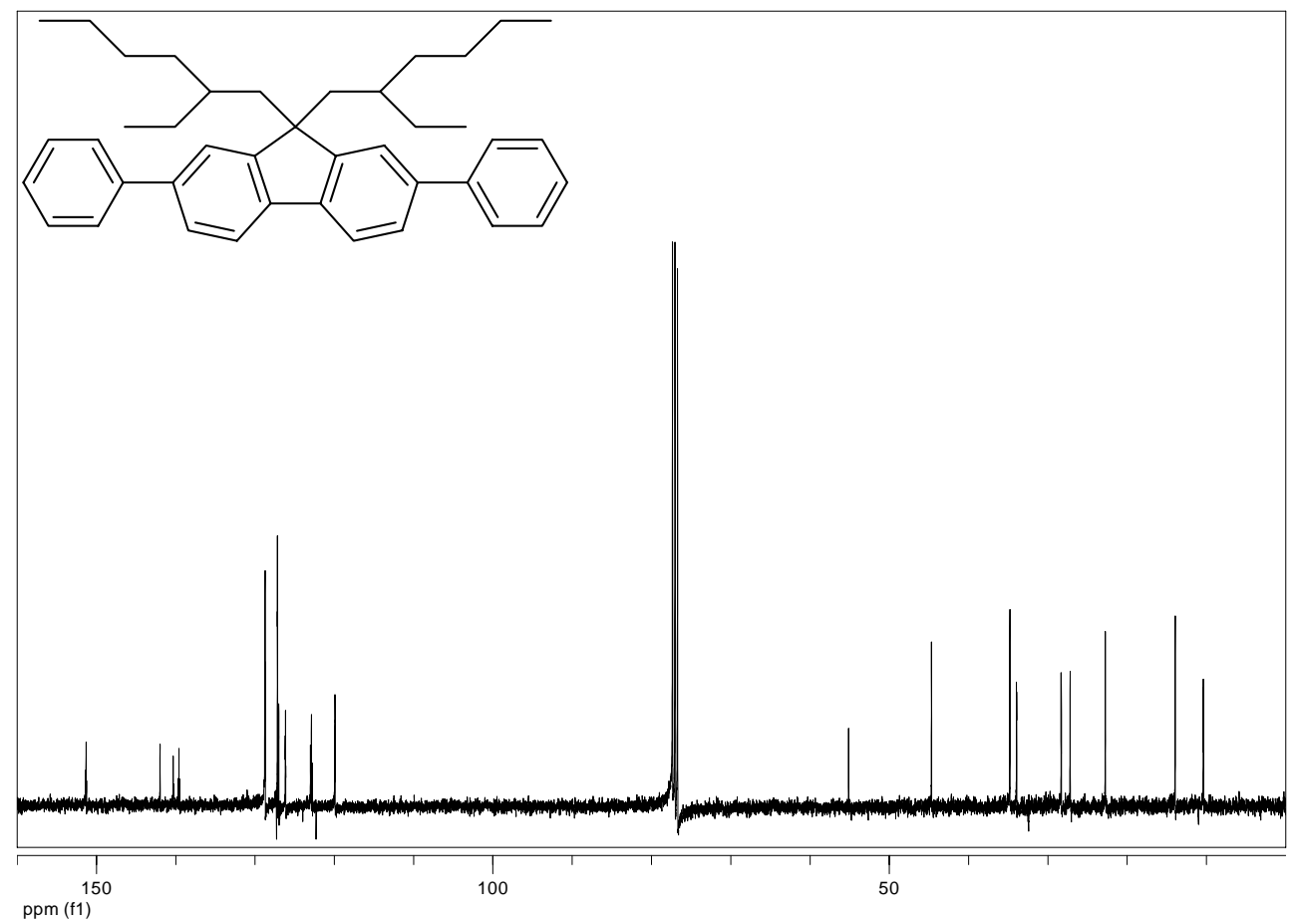

Figure $9-{ }^{13}$ C-NMR of 2,7-Bisphenyl-9,9'-bis(2-ethylhexyl)fluorene (12a). 
4.4 2,7-Bis(4-tert-butylphenyl)-9,9'-bis(2-ethylhexyl)fluorene (12b, (Table 1, Entry 2)
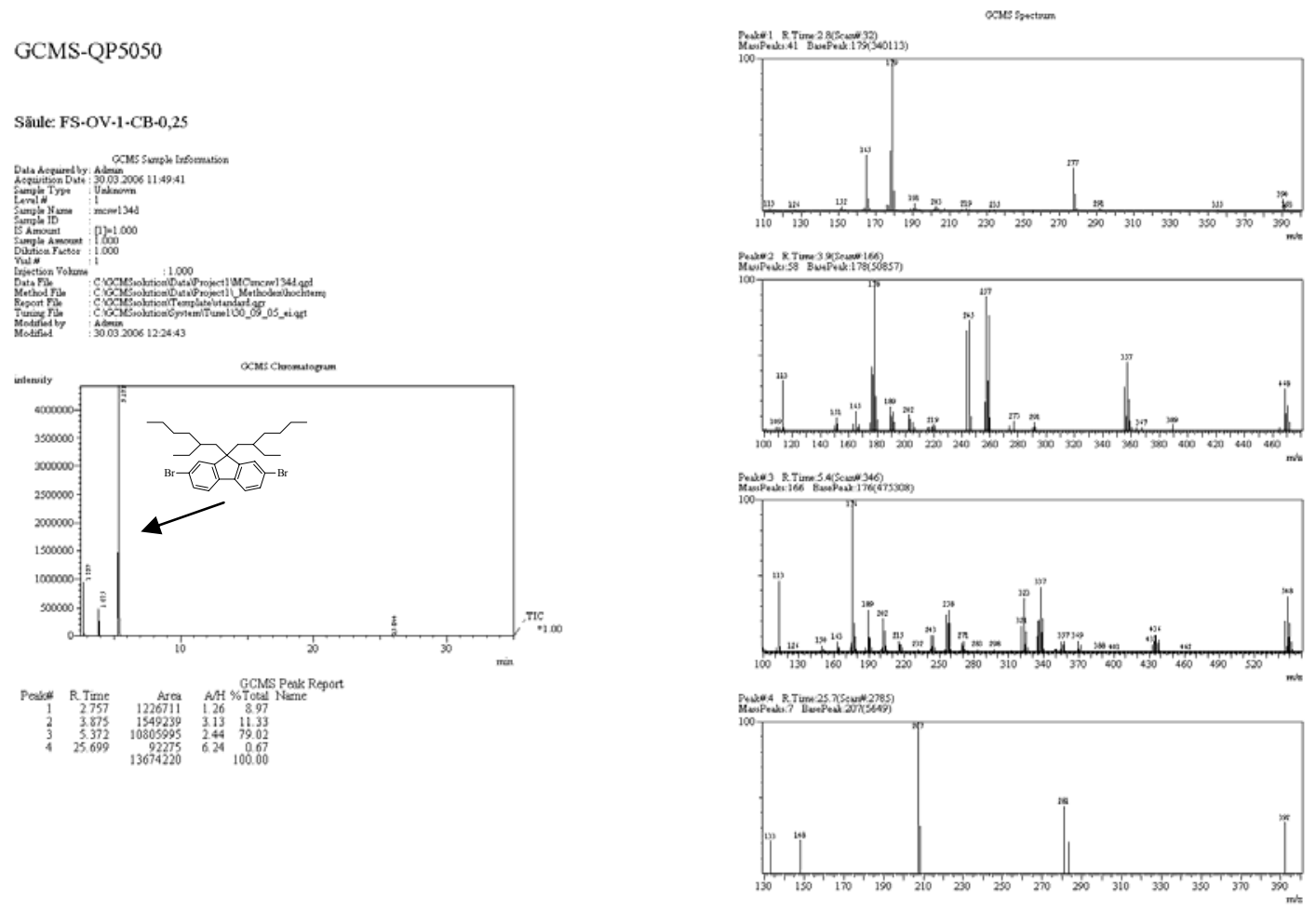

Figure 10 - GC-MS spectra of entry 2 (4a). 


\subsection{2,7-Diphenyl-9,9'-bis(2-ethylhexyl)fluorene (12a, Table 1, Entry 3)}
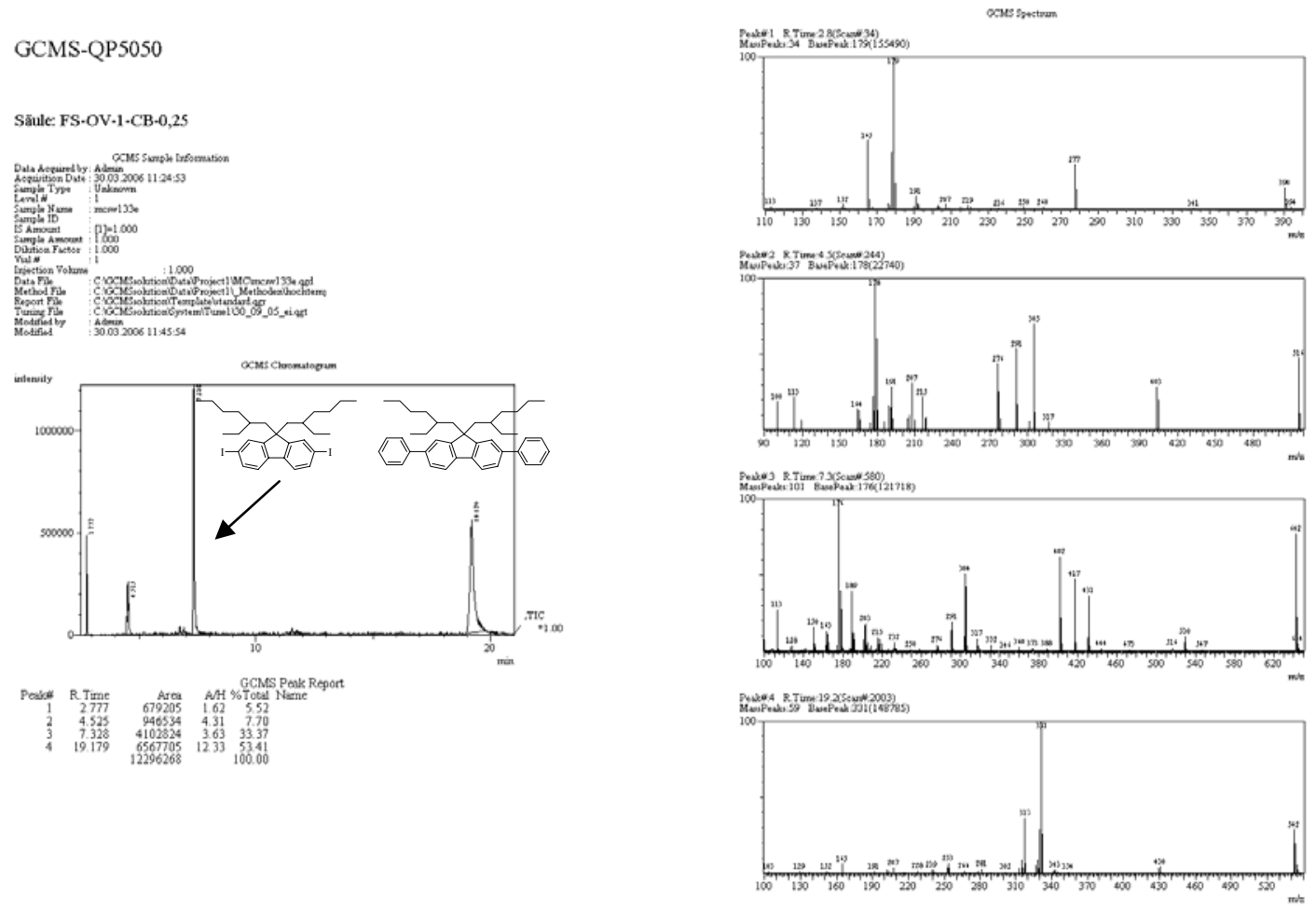

Figure 11 - GC-MS spectra of entry 3 (Mixture of 4, $X=I$ and 12a).

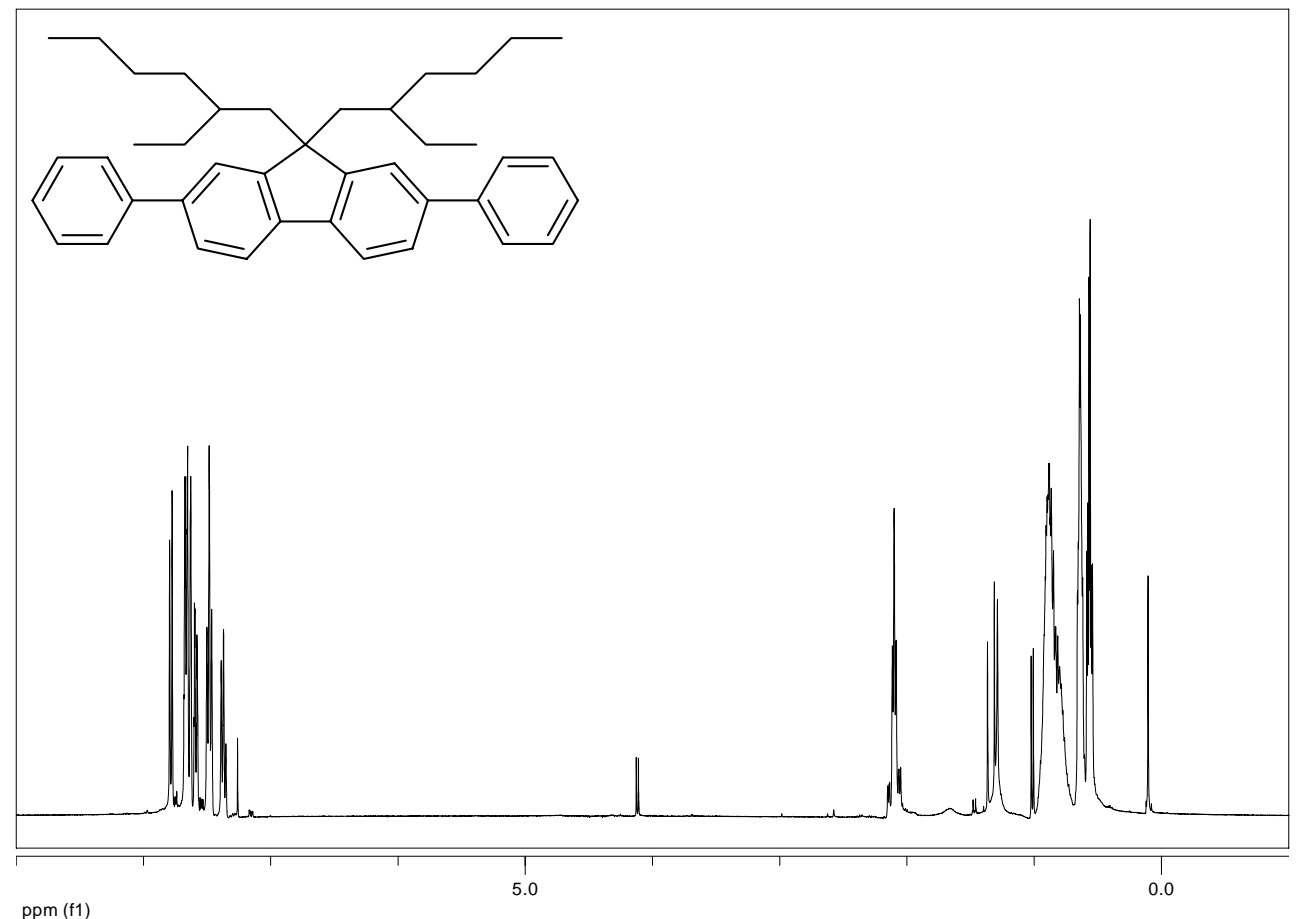

Figure 12 - ${ }^{1}$ H-NMR of 2,7-Diphenyl-9,9'-bis(2-ethylhexyl)fluorene (12a). 


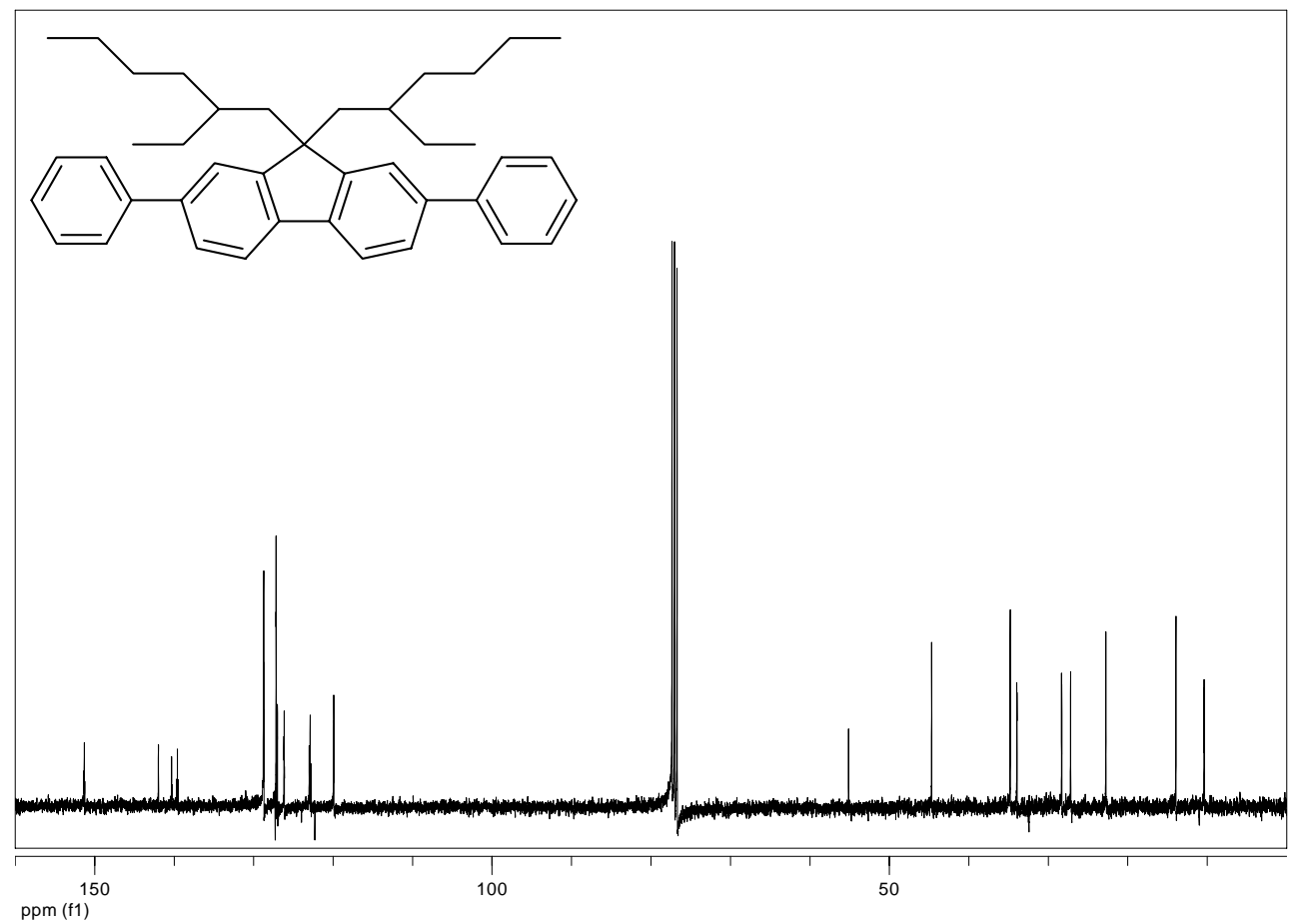

Figure 13 - ${ }^{13}$ C-NMR of 2,7-Diphenyl-9,9'-bis(2-ethylhexyl)fluorene (12a). 
4.6 2,7-Bis(4-tert-butylphenyl)-9,9'-bis(2-ethylhexyl)fluorene (12b, Table 1, Entry 4)

GCMS-QP5050

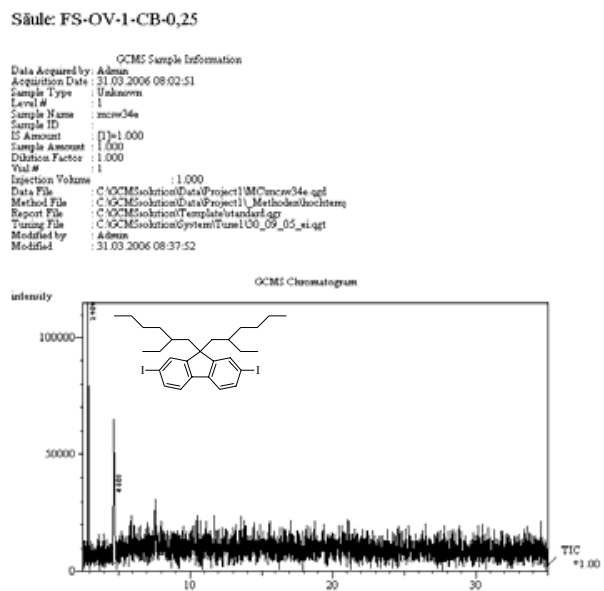

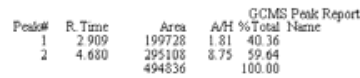
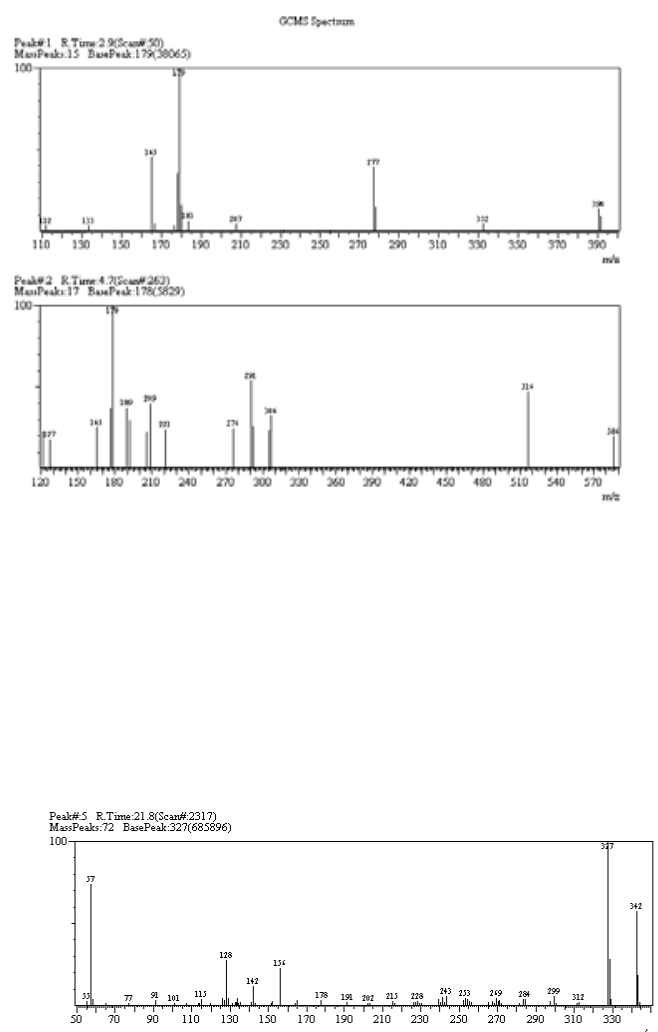

Figure 14 - GC-MS spectra of entry 4 (4b). 